\title{
Spin pump in the presence of a superconducting lead
}

\author{
Yanxia Xing, ${ }^{1}$ Bin Wang, ${ }^{1}$ Yadong Wei, ${ }^{2}$ Baigeng Wang, ${ }^{3}$ and Jian Wang ${ }^{1,4, *}$ \\ ${ }^{1}$ Key Laboratory of Materials Physics, Institute of Solid State Physics, Chinese Academy of Sciences, Hefei, Anhui, \\ People's Republic of China \\ ${ }^{2}$ Department of Physics, College of Science, Shenzhen University, Shenzhen 518060, People's Republic of China \\ ${ }^{3}$ National Laboratory of Solid State Microstructures and Department of Physics, Nanjing University, Nanjing, \\ People's Republic of China \\ ${ }^{4}$ Department of Physics, The University of Hong Kong, Pokfulam Road, Hong Kong, People's Republic of China
} (Received 31 August 2004; revised manuscript received 18 October 2004; published 30 December 2004)

\begin{abstract}
We present a theoretical analysis of a spin pump in the presence of a superconducting lead. The spin pump is facilitated by a rotating magnetic field which provides a spin flip mechanism and hence can generate a spin current without an accompanying charge current. Using a nonequilibrium Green's function method, we obtain a general solution of the pumped charge current and spin current in both the adiabatic and non-adiabatic regimes. The numerical results for the charge current and spin current are presented as we vary different system parameters such as the gate voltage, the external magnetic field, and the pumping frequency. We find that for a quantum dot with a single resonant level in line with the Fermi energy of the left normal lead, a pure spin current is generated by a rotating magnetic field at any frequency. We have identified two kinds of photonassisted processes which dominate at low pumping frequencies and high pumping frequencies, respectively.
\end{abstract}

DOI: $10.1103 /$ PhysRevB.70.245324

PACS number(s): 73.40.-c, 72.80.Ey, 73.63.-b

\section{INTRODUCTION}

The charge and spin are two basic properties of an electron. The charge degree of freedom has been fully explored in microelectronics as well as nanoelectronics. One of the key ingredients in spintronics is to use the spin flow to control the nonlinear electronic devices. ${ }^{1}$ For this reason, much research effort has been devoted to generating the pure spin current without an accompanying charge current. One way to generate the spin current in a quantum dot system is by cyclic deformation of two or more system parameters. This parametric pumping mechanism has recently attracted increasing attention in charge pumping. ${ }^{2-8}$ If the external magnetic field is applied, the pumped charge current is spin polarized. In the presence of magnetic field, electrons with different spins experience different system parameters. It is known that the magnitude as well as the direction of the pumped charge current is very sensitive to various parameters of the system such as the potential landscape of the pump,,${ }^{9,10}$ the frequency of the driving force, ${ }^{11}$ and the Fermi energy of the leads. ${ }^{12}$ Hence, it is possible that under certain conditions, the electrons with different spins flow in the opposite direction, thereby giving rise to a spin current without accompanying charge current. Indeed, several different spin pumps have been proposed along this line. For an interacting system, Sharma and Chamon studied the quantum pump for both spin and charge transport. ${ }^{13}$ For noninteracting systems, an adiabatic quantum pump was proposed ${ }^{14}$ which generates the pure spin current by cyclic variation of the confining potential of the quantum dot and the external magnetic field. In fact, this proposal has been realized experimentally ${ }^{15}$ that spin current was detected. Similar ideas have also been proposed to generate pure spin current using the Zeeman effect for a quantum dot. ${ }^{16,17}$ For instance, the spin current can be generated when the external magnetic field which induces a Zeeman energy is chosen as one of the pumping parameters. ${ }^{16}$ Due to peculiar electronic properties of the carbon nanotube (CNT), ${ }^{18-22}$ the CNT based quantum pump shows antisymmetric pumped signals near the many doubly degenerate resonant levels of the finite-length CNT for a wide range of energies. ${ }^{23}$ Because of this reversal of the pumped charge current together with the Zeeman effect, the pure spin current can be achieved for the Fermi energy near the resonant levels in the presence of a magnetic field. ${ }^{17} \mathrm{In}$ the nonadiabatic regime, using the property of charge current reversal, the spin current can be delivered from a normal quantum dot connected by two ferromagnetic leads. ${ }^{24}$ The spin current can also be produced by either a rotating magnetic moment or a rotating external magnetic field which leads to the unipole spin battery which gives constant spin current. ${ }^{25,26}$ To drive a spin current for future spintronic circuits, a spin-cell device which provides the necessary constant spin-motive force is needed. ${ }^{27,28}$ By shining microwaves on a double quantum dot in the presence of a nonuniform magnetic field, such a bipolar spin battery is proposed. ${ }^{27}$ In the presence of magnetic barriers, the spin current is found to pump out using the adiabatic theory. ${ }^{29}$ So far, the investigations on the spin current are focused on normal nanostructures. It will be interesting to study a hybrid structure with a superconducting lead where the Andreev reflection is present near the normal-superconductor (NS) interface. In this paper, we investigate a spin pump for a twodimensional quantum dot with a single level $\epsilon$ connected by a normal and a superconducting lead. For simplicity, in the following discussion we assume that $0<\epsilon<\Delta$. The spin pump is facilitated by a rotating magnetic field: $B_{0}(t)$ $=B_{0}[\sin \theta \cos \omega \mathbf{t} \mathbf{i}+\sin \theta \sin \omega t \mathbf{j}+\cos \theta \mathbf{k}]$, whose $z$ component gives the Zeeman split and whose rotating component provides a spin flip mechanism. For the spin pump with normal leads the rotating magnetic field generates an effective spin-motive force: an incoming spin up electron with energy 
below the Fermi level enters the quantum dot, absorbs a photon, jumps to the level $\epsilon$, becomes spin down due to the spin flip mechanism, and finally exits to the same lead with down spin. Hence a spin current is produced. In the presence of a superconducting lead, a second photon-assisted process can also drive the spin current. This process is effective at large frequencies $\omega>|\epsilon|+\Delta$ with $\Delta$ the superconducting gap such that electrons from the superconducting lead with energy below $-\Delta$ can also participate in the photon assisted transport. ${ }^{30}$ In this case, electrons with spin up (or down) from both leads can absorb a photon with spin flip at the same time, jump to the level $\epsilon$ (assuming $B_{z}=0$ for simplicity), and exit to the left lead. Our results show that when the resonant level of the quantum dot is in line with the Fermi level the charge current is zero and spin current is pumped out which exhibits resonance features.

The rest of this paper is organized as follows: In Sec. II, the theoretical formalisms for calculating the charge current and spin current in both adiabatic and nonadiabatic regimes are presented. In Sec. III, the numerical calculations and discussions of these results are given.

\section{THEORETICAL FORMALISM}

The system that we consider in this paper consists of a two-dimensional quantum dot in the presence of a rotating magnetic field connected by a normal lead and a superconducting lead. The Hamiltonian of the system is written as follows:

$$
H=H_{L}+H_{R}+H_{d o t}+H_{T},
$$

where $H_{L}$ describes the left normal electrode and $H_{R}$ the right superconducting electrode,

$$
\begin{gathered}
H_{L}=\sum_{k \sigma} \epsilon_{L, k} C_{L, k \sigma}^{\dagger} C_{L, k \sigma}=\sum_{k} \epsilon_{L, k} C_{L, k \uparrow}^{\dagger} C_{L, k \uparrow}+\sum_{k} \epsilon_{L, k} C_{L, k \downarrow}^{\dagger} C_{L, k \downarrow}, \\
H_{R}=\sum_{k \sigma} \epsilon_{R, k} C_{R, k \sigma}^{\dagger} C_{R, k \sigma}+\sum_{k}\left[\Delta C_{R, k \downarrow} C_{R,-k \uparrow}+\Delta C_{R,-k \uparrow}^{\dagger} C_{R, k \downarrow}^{\dagger}\right],
\end{gathered}
$$

where for simplicity we have assumed that the gap energy $\Delta$ is real. We assume that the Fermi energy of the left lead is in line with the superconducting condensate of the right lead $\mu_{s}$ which is set to zero, i.e., $E_{F}=0$. Furthermore, there is no bias applied in the left and right leads since we are interested in the pumped current. In Eq. (1), $H_{d o t}$ is the Hamiltonian of the quantum dot with a single resonant level $\epsilon$ which can be varied by applying a gate voltage. $H_{d o t}$ contains a timeindependent part $H_{d o t}^{0}$ which is a diagonal matrix (in spin space) and a time-dependent part $H^{\prime}(t)$ which is offdiagonal. Setting $\gamma=B_{0} \sin \theta$, we have

$$
H_{d o t}^{0}=\sum_{\sigma}\left[\epsilon+\sigma \mu B_{0} \cos \theta\right] d_{\sigma}^{\dagger} d_{\sigma},
$$

where $\sigma= \pm 1$ denotes the spin and

$$
H^{\prime}(t)=\gamma e^{-i \omega t} d_{\uparrow}^{\dagger} d_{\downarrow}+\gamma e^{i \omega t} d_{\downarrow}^{\dagger} d_{\uparrow}
$$

due to the rotating magnetic field. ${ }^{31}$ It is the term $H^{\prime}(t)$ that provides the spin flip mechanism and hence the total spin current is not conserved. Finally, in Eq. (1), $H_{T}$ describes the coupling between quantum dot and the electrodes,

$$
\begin{aligned}
H_{T} & =\sum_{\sigma, k, \alpha} t_{k, \alpha, \sigma} C_{\alpha, k \sigma}^{\dagger} d_{\sigma}+\text { H.c. } \\
& =\sum_{k, \alpha} t_{k, \alpha, \uparrow} C_{\alpha, k \uparrow}^{\dagger} d_{\uparrow}+t_{k, \alpha, \downarrow} C_{\alpha, k \downarrow}^{\dagger} d_{\downarrow}+\text { H.c. }
\end{aligned}
$$

Due to the spin flip mechanism introduced by the rotating magnetic field and the presence of the superconducting lead, we have to consider both electrons and holes together with their spins. For this reason, we consider the following Nambu representation: ${ }^{32}$

$$
\Psi_{\alpha, k}=\left(\begin{array}{c}
a_{\alpha, k 1} \\
a_{\alpha, k 2} \\
a_{\alpha, k 3} \\
a_{\alpha, k 4}
\end{array}\right) \equiv\left(\begin{array}{c}
C_{\alpha, k \uparrow} \\
C_{\alpha,-k \downarrow}^{\dagger} \\
C_{\alpha, k \downarrow} \\
C_{\alpha,-k \uparrow}^{\dagger}
\end{array}\right), \quad \Phi=\left(\begin{array}{c}
d_{\uparrow} \\
d_{\downarrow}^{\dagger} \\
d_{\downarrow} \\
d_{\uparrow}^{\dagger}
\end{array}\right) .
$$

Here the operator $C_{\alpha, k \sigma}$ destroys an electron with spin $\sigma$ and $C_{\alpha,-k \sigma}^{\dagger}$ creates an electron with spin $\sigma$ or effectively destroys a hole with an opposite spin $\bar{\sigma}$. So we have four kinds of particles: (1) electrons with spin up; (2) holes with spin up; (3) electrons with spin down; and (4) holes with spin down. With this representation, the Hamiltonian Eq. (1) can be expressed in a matrix form:

$$
\begin{gathered}
H_{L}=\sum_{k} \Psi_{L, k}^{\dagger}\left(\begin{array}{cccc}
\epsilon_{L, k} & 0 & 0 & 0 \\
0 & -\epsilon_{L,-k} & 0 & 0 \\
0 & 0 & \epsilon_{L, k} & 0 \\
0 & 0 & 0 & -\epsilon_{L,-k}
\end{array}\right) \Psi_{L, k}, \\
H_{R}=\sum_{k} \Psi_{R, k}^{\dagger}\left(\begin{array}{cccc}
\epsilon_{R, k} & \Delta & 0 & 0 \\
\Delta & -\epsilon_{R,-k} & 0 & 0 \\
0 & 0 & \epsilon_{R, k} & -\Delta \\
0 & 0 & -\Delta & -\epsilon_{R,-k}
\end{array}\right) \Psi_{R, k}, \\
H_{d o t}=H_{d o t}^{0}+H^{\prime}(t),
\end{gathered}
$$

with

$$
H_{d o t}^{0}=\Phi^{\dagger}\left(\begin{array}{cccc}
\epsilon_{1} & 0 & 0 & 0 \\
0 & -\epsilon_{2} & 0 & 0 \\
0 & 0 & \epsilon_{2} & 0 \\
0 & 0 & 0 & -\epsilon_{1}
\end{array}\right) \Phi
$$

where $\epsilon_{1,2}=\epsilon \pm \mu B_{0} \cos \theta$ and

$$
H^{\prime}(t)=\Phi^{\dagger}\left(\begin{array}{cccc}
0 & 0 & \gamma e^{-i \omega t} & 0 \\
0 & 0 & 0 & -\gamma e^{-i \omega t} \\
\gamma e^{i \omega t} & 0 & 0 & 0 \\
0 & -\gamma e^{i \omega t} & 0 & 0
\end{array}\right) \Phi
$$




$$
H_{T}=\sum_{k, \alpha} \Psi_{k, \alpha}^{\dagger}\left(\begin{array}{cccc}
t_{k, \alpha, \uparrow} & 0 & 0 & 0 \\
0 & -t_{-k, \alpha, \downarrow}^{*} & 0 & 0 \\
0 & 0 & t_{k, \alpha, \downarrow} & 0 \\
0 & 0 & 0 & -t_{-k, \alpha, \uparrow}^{*}
\end{array}\right) \Phi . \quad \boldsymbol{\Gamma}_{R}(E)=\theta(|E|-|\Delta|) \frac{\nu \Gamma_{R}}{\sqrt{E^{2}-\Delta^{2}}}\left(\begin{array}{cccc}
E & -\Delta & 0 & 0 \\
-\Delta & E & 0 & 0 \\
0 & 0 & E & \Delta \\
0 & 0 & \Delta & E
\end{array}\right),
$$

Note that since we have doubled the Hilbert space in a 4 $\times 4$ Nambu representation, a factor of $\frac{1}{2}$ should be included to account for this in the Hamiltonian. From now on, we will include this factor when we calculate the charge and spin current. With this Hamiltonian, we will derive in the following sections the pumped current in the adiabatic regime, where the pumping frequency is very small, and in the nonadiabatic regime where the pumping frequency is finite.

\section{A. Adiabatic regime}

In this section, we examine the pumped current in the low-frequency limit. In this limit, the system is nearly in equilibrium and we can use the equilibrium Green's function to characterize the pumping process. Using the distribution function, the total number of particles of type $n$ in the scattering system during the pumping process is given by

$$
N_{n}(x, t)=-i \int \frac{d E}{2 \pi}\left[\left[\mathbf{G}^{<}\left(E, B_{0}(t)\right)\right]_{n n}\right]_{x x},
$$

where $\mathbf{G}^{<}$is the lesser Green's function in real and space, and $x$ labels the position so that each $\mathbf{G}^{<}(x)$ is a $4 \times 4$ submatrix corresponds to the $4 \times 4$ Nambu representation. Here $n n=11,22,33$, 44 label the diagonal matrix elements which denote, respectively, the spin up electron, spin up hole, spin down electron, and spin down hole. $B_{0}(t)$ is the external parameter due to the rotating magnetic field which facilitates the pumping process. In the absence of the external bias, the equilibrium Green function $\mathbf{G}^{<}$is related to the retarded and advanced Green functions $\mathbf{G}^{r}$ and $\mathbf{G}^{a}$ as follows:

$$
\mathbf{G}^{<}\left(E, B_{0}\right)=\mathbf{G}^{r}\left(E, B_{0}\right) \mathbf{\Sigma}^{<} \mathbf{G}^{a}\left(E, B_{0}\right) .
$$

From Eqs. (13) and (14), we obtain

$$
\left[\frac{d N_{\alpha \sigma}(t)}{d t}\right]_{n n}=-\int \frac{d E}{2 \pi}\left(-\partial_{E} f\right) \operatorname{Tr}_{o}\left[\boldsymbol{\Gamma}_{\alpha} \mathbf{G}^{r}(t) \frac{d \mathbf{H}^{\prime}}{d t} \mathbf{G}^{a}(t)\right]_{n n},
$$

where the effective linewidth functions are

$$
\boldsymbol{\Gamma}(E)=\boldsymbol{\Gamma}_{L}(E)+\boldsymbol{\Gamma}_{R}(E)
$$

with

$$
\Gamma_{L}(E)=\Gamma_{L}\left(\begin{array}{cccc}
1 & 0 & 0 & 0 \\
0 & 1 & 0 & 0 \\
0 & 0 & 1 & 0 \\
0 & 0 & 0 & 1
\end{array}\right)
$$

and where $\nu=1$ for $E>-\Delta$ and $\nu=-1$ otherwise.

In the adiabatic limit, the Green's function is written as

$$
\left[\mathbf{G}^{r}(t)\right]^{-1}=\left(\begin{array}{cccc}
-\epsilon+\frac{i}{2} \Gamma & -\frac{\Gamma}{2} & -\gamma e^{-i \omega t} & 0 \\
-\frac{\Gamma}{2} & \epsilon+\frac{i}{2} \Gamma & 0 & \gamma e^{-i \omega t} \\
-\gamma e^{i \omega t} & 0 & -\epsilon+\frac{i}{2} \Gamma & \frac{\Gamma}{2} \\
0 & \gamma e^{i \omega t} & \frac{\Gamma}{2} & \epsilon+\frac{i}{2} \Gamma
\end{array}\right),
$$

where we have set $\theta=\pi / 2$ for simplicity and $\Gamma=\Gamma_{L}+\Gamma_{R}$. Since the electrons and holes have opposite charge, we obtain, from Eqs. (15) and (19), the charge current $J_{e \alpha}$ and spin current $J_{s \alpha}$ through $\alpha$ electrode in the adiabatic regime,

$$
J_{e \alpha}=\frac{1}{2} \operatorname{Tr}_{s}\left[\sigma_{e} d N_{\alpha \sigma}(t) / d t\right]=0, \quad J_{s R}=0,
$$

and

$J_{s L}=\frac{1}{2} \operatorname{Tr}_{s}\left[\sigma_{s} d N_{L \sigma}(t) / d t\right]=\frac{\hbar}{2} \frac{2 \omega B_{0}{ }^{2} \Gamma \Gamma_{L}}{2 \pi\left[\left(\epsilon^{2}+\Gamma^{2} / 2-B_{0}{ }^{2}\right)^{2}+\Gamma^{2} B_{0}{ }^{2}\right]}$,

where the trace $\operatorname{Tr}_{s}$ is over the spin space and $\operatorname{Tr}_{o}$ is over orbital degrees of freedom. Here $\sigma_{e}$ and $\sigma_{s}$ are defined as follows:

$$
\begin{aligned}
\sigma_{e} & =q\left(\begin{array}{cccc}
1 & 0 & 0 & 0 \\
0 & -1 & 0 & 0 \\
0 & 0 & 1 & 0 \\
0 & 0 & 0 & -1
\end{array}\right), \\
\sigma_{s} & =\frac{\hbar}{2}\left(\begin{array}{cccc}
1 & 0 & 0 & 0 \\
0 & 1 & 0 & 0 \\
0 & 0 & -1 & 0 \\
0 & 0 & 0 & -1
\end{array}\right) .
\end{aligned}
$$

Since we have set the Fermi level of the left lead to zero, the gap energy $\Delta$ does not appear in the expression of spin current. We note that in the absence of the superconducting lead, the spin current in the adiabatic regime is ${ }^{26}$

$$
J_{s L}=\frac{\hbar}{2} \frac{2 \omega B_{0}{ }^{2} \Gamma \Gamma_{L}}{2 \pi\left[\left(\epsilon^{2}+\Gamma^{2} / 4-B_{0}{ }^{2}\right)^{2}+\Gamma^{2} B_{0}{ }^{2}\right]} .
$$

We see that the difference between Eqs. (21) and (22) is the factor of 2 of $\Gamma^{2}$ in the denominator. As a result, the peak positions of spin current are different with or without the 
superconducting lead. Since there is no electric current in the normal lead, the supercurrent is zero in the superconducting lead from the current conservation. As a result, there is no Andreev reflection in the adiabatic regime. Hence the enhancement of the charge pumping ${ }^{33}$ due to the Andreev reflection is absent as can be seen from Eqs. (21) and (22).

\section{B. Nonadiabatic regime}

Going beyond the adiabatic approximation, the charge and spin current can be calculated analytically using the nonequilibrium Green's function (NEGF) method. To do that, it is convenient to define the particle current operator in spin space,

$$
I_{\alpha n}=d N_{\alpha n} / d t,
$$

where $N_{\alpha n}=\Sigma_{k} a_{\alpha k n}^{\dagger} a_{\alpha k n}$ with $a_{\alpha k n}$ defined in Eq. (6). With this definition, the charge current and spin current are written as

$$
J_{\alpha}^{e}(t)=\frac{1}{2} \operatorname{Tr}_{s}\left[\sigma_{e} d N_{\alpha} / d t\right]=-\frac{1}{2} \sum_{k} \operatorname{Tr}_{s}\left[\sigma_{e}\left(T_{k \alpha} \mathbf{G}_{d, k \alpha}^{<}(t, t)+\text { H.c. }\right)\right]
$$

and

$$
\begin{aligned}
J_{\alpha}^{s}(t) & =\frac{1}{2} \operatorname{Tr}_{s}\left[\sigma_{s} d N_{\alpha} / d t\right] \\
& =-\frac{1}{2} \sum_{k} \operatorname{Tr}_{s}\left[\sigma_{s}\left(T_{k \alpha} \mathbf{G}_{d, k \alpha}^{<}(t, t)+\text { H.c. }\right)\right] .
\end{aligned}
$$

Using the theorem of analytic continuation, Eqs. (24) and (25) become

$$
\begin{aligned}
J_{\alpha}^{e, s}(t)= & -\frac{1}{2} \int d t_{1} \operatorname{Tr}_{s}\left[\sigma _ { e , s } \operatorname { T r } _ { o } \left(\mathbf{G}^{r}\left(t, t_{1}\right) \Sigma_{\alpha}^{<}\left(t_{1}-t\right)\right.\right. \\
& \left.\left.+\mathbf{G}^{<}\left(t, t_{1}\right) \Sigma_{\alpha}^{a}\left(t_{1}-t\right)+\text { H.c. }\right)\right],
\end{aligned}
$$

where $G^{r}\left(t, t_{1}\right)$ and $G^{<}\left(t, t_{1}\right)$ are the Green's function of the quantum dot. The effect of electrodes has been included in the self-energy $\Sigma^{\gamma}(\gamma=r, a,<)$. Now we consider the dc components, the averaged current flowing through contact $\alpha$ due to the rotating magnetic field $H^{\prime}(t)$ in one period $\tau$ is given by

$$
J_{\alpha}^{e, s}=\frac{1}{\tau} \int_{0}^{\tau} d t J_{\alpha}^{e, s}(t)=\frac{1}{2 L} \int_{-L}^{L} d t J_{\alpha}^{e, s}(t),
$$

where $L=N \tau$ and we will take the limit $N \rightarrow \infty$. From Eqs. (26) and (27), we obtain

$$
\begin{aligned}
J_{\alpha}^{e, s}= & -\frac{1}{4 L} \int d t d t_{1} \operatorname{Tr}_{s}\left[\sigma _ { e , s } \operatorname { T r } _ { o } \left(G^{r}\left(t, t_{1}\right) \Sigma_{\alpha}^{<}\left(t_{1}-t\right)\right.\right. \\
& \left.\left.+G^{<}\left(t, t_{1}\right) \Sigma_{\alpha}^{a}\left(t_{1}-t\right)+\text { H.c. }\right)\right] .
\end{aligned}
$$

Carrying out the following double Fourier transform over time $t$ and $t^{\prime}$,

$$
\mathbf{G}^{\gamma}\left(E, E^{\prime}\right)=\int d t d t^{\prime} e^{i E t-i E^{\prime} t^{\prime}} \mathbf{G}^{\gamma}\left(t, t^{\prime}\right)
$$

with $\gamma=r, a,<$, Eq. (28) becomes

$$
\begin{aligned}
J_{\alpha}^{e, s}= & -\frac{1}{2 L} \int \frac{d E}{2 \pi} \operatorname{Tr}_{s}\left[\sigma _ { e , s } \operatorname { T r } _ { o } \left(G^{r}(E, E) \Sigma_{\alpha}^{<}(E)\right.\right. \\
& \left.\left.-\Sigma_{\alpha}^{<}(E) G^{a}(E, E)+G^{<}(E, E) \Sigma_{\alpha}^{a}(E)-\Sigma_{\alpha}^{r}(E) G^{<}(E, E)\right)\right] .
\end{aligned}
$$

In Eq. (30) the Green functions $G^{r, a}$ and $G^{<}$are calculated from the following Dyson equation:

$$
\mathbf{G}^{r}\left(t, t^{\prime}\right)=\mathbf{G}^{r 0}\left(t-t^{\prime}\right)+\int d t_{1} \mathbf{G}^{r}\left(t, t_{1}\right) H^{\prime}\left(t_{1}\right) \mathbf{G}^{r 0}\left(t_{1}-t^{\prime}\right)
$$

and the Keldysh equation

$$
\mathbf{G}^{<}\left(t, t^{\prime}\right)=\int d t_{1} d t_{2} \mathbf{G}^{r}\left(t, t_{1}\right) \mathbf{\Sigma}^{<}\left(t_{1}-t_{2}\right) \mathbf{G}^{a}\left(t_{2}, t^{\prime}\right),
$$

where $\mathbf{G}^{r 0}\left(t, t^{\prime}\right)$ is the equilibrium Green function which depends only on the time difference $t-t^{\prime}$.

After the double Fourier transform over time, we write all matrix elements of $\mathbf{G}^{r}$ appearing in Eq. (31) as

$$
\begin{aligned}
\mathbf{G}_{11}^{r}\left(E, E^{\prime}\right)= & \delta\left(E-E^{\prime}\right) \mathbf{G}_{11}^{r 0}(E)+B_{0} \mathbf{G}_{13}^{r}\left(E, E^{\prime}-\omega\right) \mathbf{G}_{11}^{r 0}\left(E^{\prime}\right) \\
& -B_{0} \mathbf{G}_{14}^{r}\left(E, E^{\prime}-\omega\right) \mathbf{G}_{21}^{r 0}\left(E^{\prime}\right), \\
\mathbf{G}_{12}^{r}\left(E, E^{\prime}\right)= & \delta\left(E-E^{\prime}\right) \mathbf{G}_{12}^{r 0}(E)+B_{0} \mathbf{G}_{13}^{r}\left(E, E^{\prime}-\omega\right) \mathbf{G}_{12}^{r 0}\left(E^{\prime}\right) \\
& -B_{0} \mathbf{G}_{14}^{r}\left(E, E^{\prime}-\omega\right) \mathbf{G}_{22}^{r 0}\left(E^{\prime}\right), \\
\mathbf{G}_{13}^{r}\left(E, E^{\prime}\right)= & B_{0} \mathbf{G}_{11}^{r}\left(E, E^{\prime}+\omega\right) \mathbf{G}_{33}^{r 0}\left(E^{\prime}\right) \\
& -B_{0} \mathbf{G}_{12}^{r}\left(E, E^{\prime}+\omega\right) \mathbf{G}_{43}^{r 0}\left(E^{\prime}\right) \\
\mathbf{G}_{14}^{r}\left(E, E^{\prime}\right)= & B_{0} \mathbf{G}_{11}^{r}\left(E, E^{\prime}+\omega\right) \mathbf{G}_{34}^{r 0}\left(E^{\prime}\right) \\
& -B_{0} \mathbf{G}_{12}^{r}\left(E, E^{\prime}+\omega\right) \mathbf{G}_{44}^{r 0}\left(E^{\prime}\right) .
\end{aligned}
$$

Shifting the variable $E^{\prime}$ to $E^{\prime}-\omega$ in Eqs. (35) and (36) and then substituting into Eqs. (33) and (34), we can solve for $G_{i j}^{r}\left(E, E^{\prime}\right)$ with $i=1$ and $j=1,2,3,4$. The other matrix elements of $G_{i j}^{r}$ can be calculated in a similar way. We wish to mention that the rotating magnetic field facilitates the solution in terms of the matrix Green's functions, which otherwise would lead to equations that do not close. Once the $G^{r, a}$ is calculated the lesser Green's function is obtained from the Keldysh equation,

$$
\mathbf{G}^{<}\left(E, E^{\prime}\right)=\int \frac{d E_{1}}{2 \pi} \mathbf{G}^{r}\left(E, E_{1}\right) \mathbf{\Sigma}^{<}\left(E_{1}\right) \mathbf{G}^{a}\left(E_{1}, E^{\prime}\right) .
$$

Having obtained $\mathbf{G}^{r, a}, \mathbf{G}^{<}$, and $\boldsymbol{\Sigma}^{<, r, a}$, the charge current and spin current can be calculated from Eq. (30).

We set $\mathbf{G}^{r}(E, E+\sigma \omega)=\mathbf{G}_{\sigma}$ and $\mathbf{F}(E+\sigma \omega)=\mathbf{F}_{\sigma}$, where $F$ could be the coupling constant $\Gamma$ or Fermi distribution function $f$. For instance, $\Gamma_{\uparrow, 11}(E)=\Gamma_{11}(E+\omega)$. We write the charge and spin current as follows:

$$
\begin{aligned}
& J_{\alpha}^{e}=q\left(J_{\alpha, \uparrow}+J_{\alpha, \downarrow}\right), \\
& J_{\alpha}^{s}=\frac{1}{2}\left(J_{\alpha, \uparrow}-J_{\alpha, \downarrow}\right),
\end{aligned}
$$

where $\alpha=L, R$ and 


$$
J_{\alpha, \sigma}=\int \frac{d E}{2 \pi} T_{\alpha, \sigma}\left(f_{\sigma}-f\right) .
$$

Here

$$
T_{L, \uparrow}=\Gamma_{L, 11}\left[\Gamma_{\uparrow 33}\left(\left|\mathbf{G}_{\uparrow 13}^{r}\right|^{2}+\left|\mathbf{G}_{\uparrow 14}^{r}\right|^{2}\right)+2 \Gamma_{\uparrow 34} \operatorname{Re}\left(\mathbf{G}_{\uparrow 13}^{r} \mathbf{G}_{\uparrow 14}^{r, *}\right)\right]
$$

and

$$
\begin{aligned}
T_{R, \uparrow}= & \Gamma_{R, 11}\left[\Gamma_{\uparrow 33}\left(\left|\mathbf{G}_{\uparrow 13}^{r}\right|^{2}+\left|\mathbf{G}_{\uparrow 14}^{r}\right|^{2}\right)+2 \Gamma_{\uparrow 34} \operatorname{Re}\left(\mathbf{G}_{\uparrow 13}^{r} \mathbf{G}_{\uparrow 14}^{r, *}\right)\right] \\
& +\Gamma_{R, 21}\left[\Gamma_{\uparrow 33}\left(\mathbf{G}_{\uparrow 13}^{r} \mathbf{G}_{\uparrow 23}^{r, *}+\mathbf{G}_{\uparrow 14}^{r} \mathbf{G}_{\uparrow 24}^{r, *}\right)+\Gamma_{\uparrow 34}\left(\mathbf{G}_{\uparrow 13}^{r} \mathbf{G}_{\uparrow 24}^{r, *}\right.\right. \\
& \left.\left.+\mathbf{G}_{\uparrow 14}^{r} \mathbf{G}_{\uparrow 23}^{r, *}\right)\right]-\Sigma_{12, R}^{r}\left[\Gamma_{\uparrow 33} \operatorname{Im}\left(\mathbf{G}_{\uparrow 13}^{r} \mathbf{G}_{\uparrow 23}^{r, *}+\mathbf{G}_{\uparrow 14}^{r} \mathbf{G}_{\uparrow 24}^{r, *}\right)\right. \\
& \left.+\Gamma_{\uparrow 34} \operatorname{Im}\left(\mathbf{G}_{\uparrow 13}^{r} \mathbf{G}_{\uparrow 24}^{r, *}+\mathbf{G}_{\uparrow 14}^{r} \mathbf{G}_{\uparrow 23}^{r, *}\right)\right],
\end{aligned}
$$

where we have used the fact that $\Gamma_{\uparrow 33}=\Gamma_{\uparrow 44}$ and $\Gamma_{\uparrow 34}=\Gamma_{\uparrow 43}$ where $\Gamma_{i j}$ is the matrix element of $\boldsymbol{\Gamma}$. To obtain the current $J_{\alpha \downarrow}$ from Eqs. (40) and (41), we note that the transformation $\uparrow \rightarrow \downarrow$ implies $1 \rightarrow 3,3 \rightarrow 1,2 \rightarrow 4$, and $4 \rightarrow 2$ in the Green's function from Eq. (6). For instance, under this transformation, $G_{\uparrow 23}^{r} \rightarrow G_{\downarrow 41}^{r}$. For simplicity, in the following discussion we will consider the symmetric situation so that $t_{k L}=t_{k R}$ and let $\Gamma_{L}=\Gamma_{R}=\Gamma_{0}$. The self-energies due to the lead are

$$
\begin{gathered}
\boldsymbol{\Sigma}^{a, r,<}(E)=\boldsymbol{\Sigma}_{L}^{a, r,<}(E)+\boldsymbol{\Sigma}_{R}^{a, r,<}(E), \\
\boldsymbol{\Sigma}_{L, R}^{<}(E)=\left(\boldsymbol{\Sigma}_{L, R}^{a}(E)-\boldsymbol{\Sigma}_{L, R}^{r}(E)\right) f(E),
\end{gathered}
$$

where $f(E)$ is Fermi distribution function, and ${ }^{32}$

$$
\begin{gathered}
\boldsymbol{\Sigma}_{L}^{r}(E)=\frac{-i \Gamma_{0}}{2}\left(\begin{array}{cccc}
1 & 0 & 0 & 0 \\
0 & 1 & 0 & 0 \\
0 & 0 & 1 & 0 \\
0 & 0 & 0 & 1
\end{array}\right), \\
\boldsymbol{\Sigma}_{R}^{r}(E)=\frac{-i}{2} \frac{\nu \Gamma_{0}}{\sqrt{E^{2}-\Delta^{2}}}\left(\begin{array}{cccc}
E & -\Delta & 0 & 0 \\
-\Delta & E & 0 & 0 \\
0 & 0 & E & \Delta \\
0 & 0 & \Delta & E
\end{array}\right) .
\end{gathered}
$$

From Eqs. (43)-(45), we have

$$
\begin{gathered}
\boldsymbol{\Sigma}_{L}^{<}(E)=i \Gamma_{0} f(E)\left(\begin{array}{cccc}
1 & 0 & 0 & 0 \\
0 & 1 & 0 & 0 \\
0 & 0 & 1 & 0 \\
0 & 0 & 0 & 1
\end{array}\right), \\
\boldsymbol{\Sigma}_{R}^{<}(E)=i \theta(|E|-|\Delta|) f(E) \frac{\nu \Gamma_{0}}{\sqrt{E^{2}-\Delta^{2}}}\left(\begin{array}{ccccc}
E & -\Delta & 0 & 0 \\
-\Delta & E & 0 & 0 \\
0 & 0 & E & \Delta \\
0 & 0 & \Delta & E
\end{array}\right) .
\end{gathered}
$$

\section{RESULTS AND DISCUSSIONS}

Now we present the numerical results of charge current and spin current obtained from Eq. (39). In Fig. 1, we calcu-

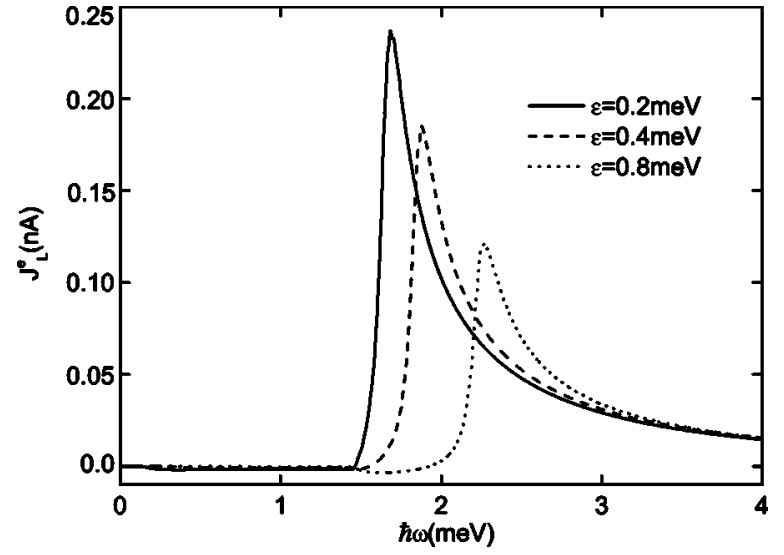

FIG. 1. The charge current $J_{L}^{e}$ vs the pumping frequency $\omega$ at different resonant energies $\epsilon: \epsilon=0.2 \mathrm{meV}$ (solid line), $\epsilon=0.4 \mathrm{meV}$ (dashed line), and $\epsilon=0.8 \mathrm{meV}$ (dotted line). Other parameters: $\mu B_{0}=0.1 \mathrm{meV}, \Gamma=0.1 \mathrm{meV}, \Delta=1.45 \mathrm{meV}$ and $\theta=\pi / 2$.

late the charge current $J_{L}^{e}$ through the left lead versus the pumping frequency $\omega$ for different resonant energies $\epsilon$ (which can be controlled by the gate voltage). ${ }^{34}$ In general, we have the following relations for the charge current, $J_{L \uparrow}^{e}$ $=J_{L \downarrow}^{h}$ and $J_{L \uparrow}^{h}=J_{L \downarrow}^{e}$. The charge current of spin up and down charged carriers are in the opposite direction. When $\epsilon=0$, the absolute values of these four terms are equal, resulting in zero charge current at any frequency. When $\epsilon$ is nonzero, there is a slight difference in charge current between up and down charged carriers for $\omega<\Delta$. Indeed, this is what we see from Fig. 1, that the charge current is small for $\omega<\Delta$. When $\omega>\Delta$, the charge currents exhibit big peaks at certain frequency $\omega_{p}$ which depends on the resonant energy $\epsilon$. For instance, we have $\omega_{p}=1.65$ and $1.94 \mathrm{meV}$ for $\epsilon=0.1$ and $0.4 \mathrm{meV}$. Our numerical results indicate that $\omega_{p}(\epsilon)$ depends on $\epsilon$ linearly.

To understand this, we discuss two physical processes that are responsible in this pumping process. Without loss of generality, we assume that $\epsilon>0$. The first process is largely responsible for the spin current. Due to the rotating magnetic field, a spin up (or down) electron near Fermi level comes from the left lead, absorbs a photon along with the spin flip and jumps to the level $\epsilon$, and finally exits to the left lead with opposite spin. This process is the dominant pumping process in the low frequency regime and gives pure spin current in the left lead with no accompanying charge current. Note that this is the only process for the normal system in the presence of rotating magnetic field as discussed in Ref. 26. As a result, the spin current shows a resonant feature with a peak near $\omega \sim \epsilon$. In the presence of the superconducting lead, there is a second pumping process at large frequencies $\omega>|\epsilon|+\Delta$ such that electrons from both leads with energy below $-\Delta$ can also participate the photon assisted transport. In this case, electrons with spin up (or down) from both leads can absorb a photon with spin flip at the same time, jump to the level $\epsilon$, and exit to the left lead. This explains why there is a peak in the left charge current and a cutoff frequency exists, $\omega_{c}$ $=|\epsilon|+\Delta$ as shown in Fig. 1 .

The spin current through the left lead vs $\omega$ is depicted in Fig. 2. For comparison, the spin current through the right 


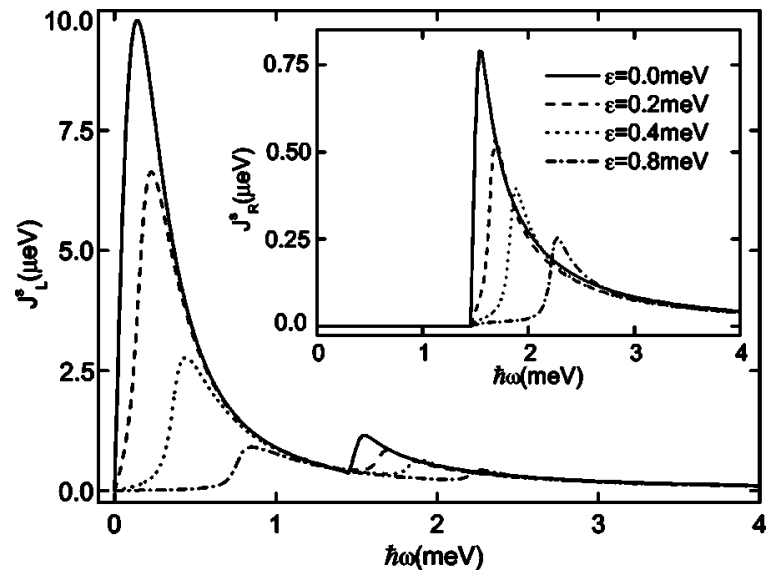

FIG. 2. The spin current of the left lead $J_{L}^{S}$ vs the pumping frequency $\omega$ at different $\epsilon$ : $\epsilon=0$ (solid line), $\epsilon=0.2 \mathrm{meV}$ (dashed line), $\epsilon=0.4 \mathrm{meV}$ (dotted line), and $\epsilon=0.8 \mathrm{meV}$ (dash-dotted line). Other parameters are the same as Fig. 1. Inset: the right spin current $J_{R}^{S}$ vs the pumping frequency $\omega$ at different $\epsilon$. The symbols are the same as the main panel.

lead vs $\omega$ is also plotted in the inset of Fig. 2. Although the charge current is nonzero when $\omega<\Delta$, the spin current in the superconducting lead must be zero due to the opposite momenta of the Cooper pair. It is precisely what we have obtained. From the inset of Fig. 2 we see that only when $\omega$ $>\Delta$, the spin current in the superconducting lead starts to occur and shows similar features as that of the charge current (see Fig. 1). As described in the first pumping process, due to the spin flip mechanism introduced by the rotating magnetic field, the incoming spin up electron (hole) is reflected back as the spin down electron (hole) so that the spin currents are in the same direction. As a result, the spin current of the left lead is much larger when $\omega<\Delta$ and shows a large peak at a certain frequency which depends on the resonant energy $\epsilon$. When the frequency is larger than the gap energy, a second small peak emerges for the spin current of the left lead.

In Fig. 3, we plot the charge current versus the pumping frequency at different magnetic fields. We see that as the

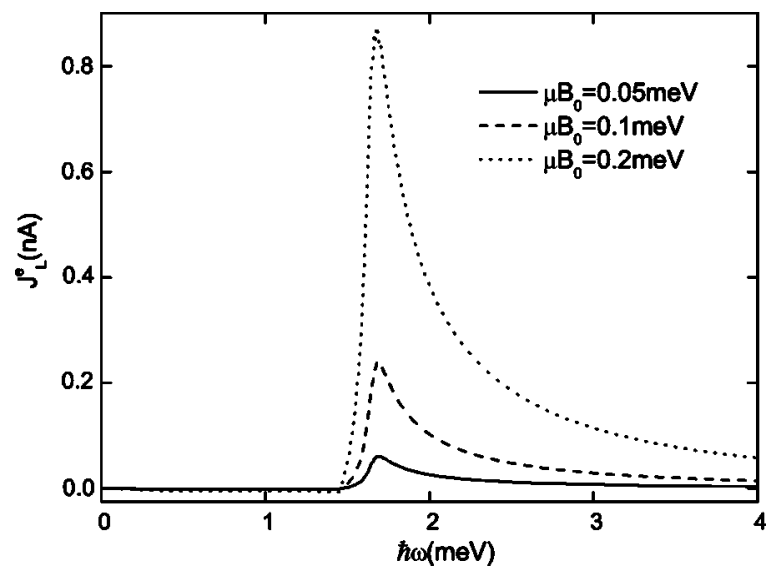

FIG. 3. The charge current $J_{L}^{e}$ vs the pumping frequency $\omega$ at different magnetic fields $\mu B_{0}: \mu B_{0}=0.05 \mathrm{meV}$ (solid line), $\mu B_{0}$ $=0.1 \mathrm{meV}$ (dashed line) and $\mu B_{0}=0.2 \mathrm{meV}$ (dotted line). Other parameters: $\epsilon=0.2 \mathrm{meV}, \Gamma=0.1 \mathrm{meV}, \Delta=1.45 \mathrm{meV}$, and $\theta=0.5 \pi$.

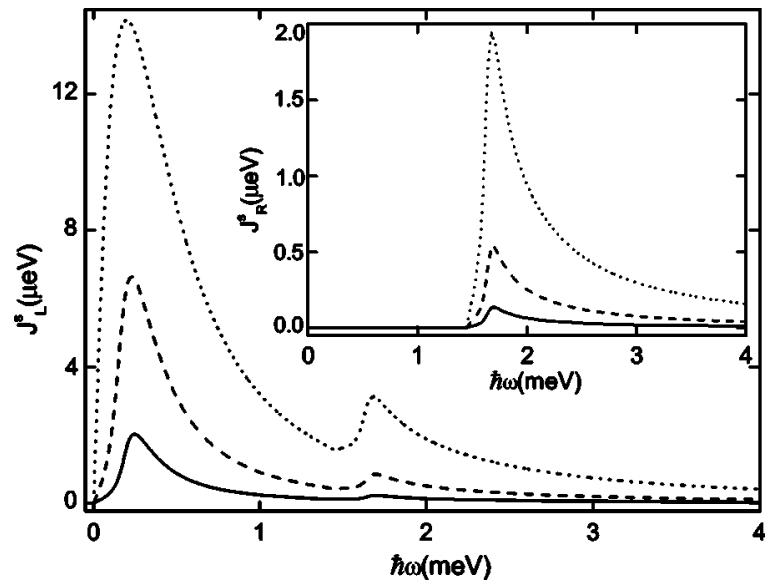

FIG. 4. The left spin current $J_{L}^{S}$ vs $\omega$ at different magnetic fields $\mu B_{0}: \mu B_{0}=0.05 \mathrm{meV}$ (solid line), $\mu B_{0}=0.1 \mathrm{meV}$ (dashed line), and $\mu B_{0}=0.2 \mathrm{meV}$ (dotted line). Other parameters are the same as Fig. 3. Inset: the right spin current $J_{R}^{S}$ vs $\omega$ at different magnetic fields. The symbols are the same as the main panel.

magnetic field is increased the charge current increases. The resonant feature is similar to that of Fig. 1 except that the peak positions do not shift. This is understandable since the resonant level $\epsilon$ remains unchanged.

The spin current of the left lead versus the pumping frequency is plotted in Fig. 4 and the corresponding spin current of the right lead is shown in the inset. For the left lead, the spin current exhibits a double peak structure while for the right lead the spin current shows a single peak. As the magnetic field is increased the spin current increases but the peak positions do not change. Clearly, the physics of Fig. 4 is similar to that of Fig. 2 and can be understood within the two pumping processes discussed above.

In the inset of Fig. 5, we plot the charge current vs $\epsilon$ for two different pumping frequencies: (1). $\omega<\Delta$ (the left inset) and (2). $\omega>\Delta$ (the right inset). Two points should be mentioned here. First, the charge current can change its direction when the resonant level (which can be controlled by the gate voltage) crosses zero. Second, when $\epsilon$ is nonzero, the charge current can also change its sign by changing the pumping frequency (compare the left inset with the right inset). The current reversal as $\epsilon$ changes sign can be understood as follows. When $\epsilon>0$ the charge carriers that dominant the two pumping processes discussed above are electrons. However, the holes dominant the same processes when $\epsilon<0$. Since the only difference it makes when $\epsilon$ changes sign is to change the sign of charged carriers, we have $\mathrm{e}^{35}$

$$
\begin{gathered}
J_{L}^{e}(\epsilon>0)=-J_{L}^{e}(\epsilon<0), \\
J_{L, R}^{s}(\epsilon>0)=J_{L, R}^{s}(\epsilon<0) .
\end{gathered}
$$

In the main panel of Fig. 5, we plot the charge current vs $\epsilon$ for magnetic fields. Similar current reversal behaviors are observed.

In Fig. 6, the spin currents of the left lead vs $\epsilon$ at different pumping frequencies $(\omega<\Delta)$ are plotted. When $\omega$ is small, the spin current shows clearly two symmetric peaks with 


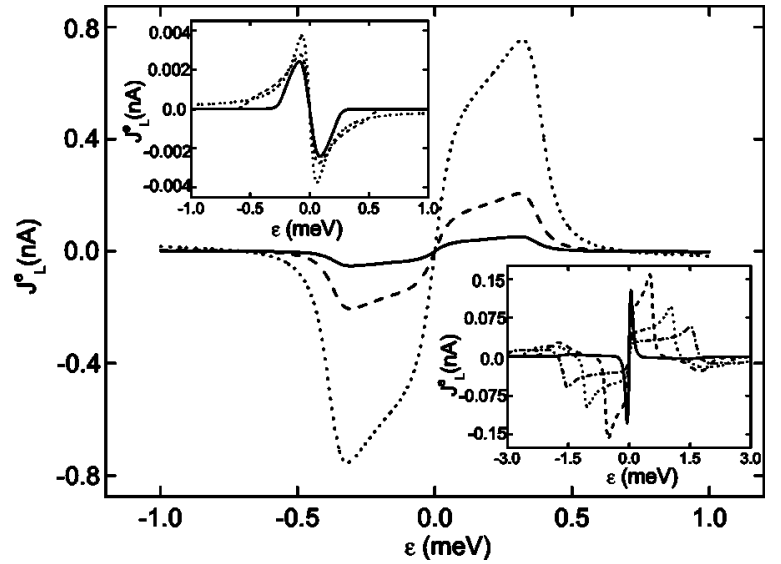

FIG. 5. The charge current $J_{L}^{e}$ vs the resonant energy $\epsilon$ at different magnetic fields: $\mu B_{0}=0.05 \mathrm{meV}$ (solid line), $\mu B_{0}=0.1 \mathrm{meV}$ (dashed line) and $\mu B_{0}=0.2 \mathrm{meV}$ (dotted line). Here $\omega=1.8 \mathrm{meV}$ and other parameters are the same as Fig. 1. The left inset: $J_{L}^{e}$ vs $\epsilon$ at different frequencies: $\omega=0.2 \mathrm{meV}$ (solid line), $\omega=0.5 \mathrm{meV}$ (dashed line), and $\omega=1.4 \mathrm{meV}$ (dotted line). The right inset: $J_{L}^{e}$ vs $\epsilon$ at much larger frequencies: $\omega=1.5 \mathrm{meV}$ (solid line), $\omega=2.0 \mathrm{meV}$ (dashed line), $\omega=2.5 \mathrm{meV}$ (dotted line), and $\omega=3.0$ (dash-dotted line). The other parameters are the same as Fig. 1.

respect to $\epsilon=0$. When $\omega$ increases, the two peaks start to merge. The double-peak structure can be understood from the expression of the pumped spin current in the adiabatic regime. The right spin current at larger frequencies $(\omega>\Delta)$ is shown in the right inset. We see that there is always a peak at $\epsilon=0$. When the frequency is slightly greater than the gap energy $\Delta$, the spin current exhibits a sharp peak near $\epsilon=0$. As the frequency increases, two sideband peaks emerge at $|\epsilon|$ $\sim \omega-\Delta$ (see dashed and dotted lines of the right inset). Obviously this sideband is due the second pumping process. When $|\epsilon|$ is greater than $\omega-\Delta$, the electron from below $-\Delta$ cannot gain enough energy by absorbing a photon of energy $\omega$ to jump to the resonant level $\epsilon$. As a result, the spin current drops drastically. At an even larger $\epsilon$, the first pumping process dominates and the spin currents show peaks near $\epsilon$ $\sim \omega$. In the left inset of Fig. 6, the left spin currents vs $\epsilon$ at different frequencies are also plotted. Here we see again the sideband structure due to the second pumping process. We also see that the spin current exhibit shoulders near $\epsilon \sim \omega$ beyond which the spin current decreases quickly. This again is attributed to the first pumping process that an electron jumps to the resonant level by photon absorption.

In summary, we have studied the spin current pumped by a rotating magnetic field for a normal superconducting hybrid system. The spin current was calculated in both adiabatic and non-adiabatic regimes using the NEGF method. Two photon assisted pumping processes are identified. One

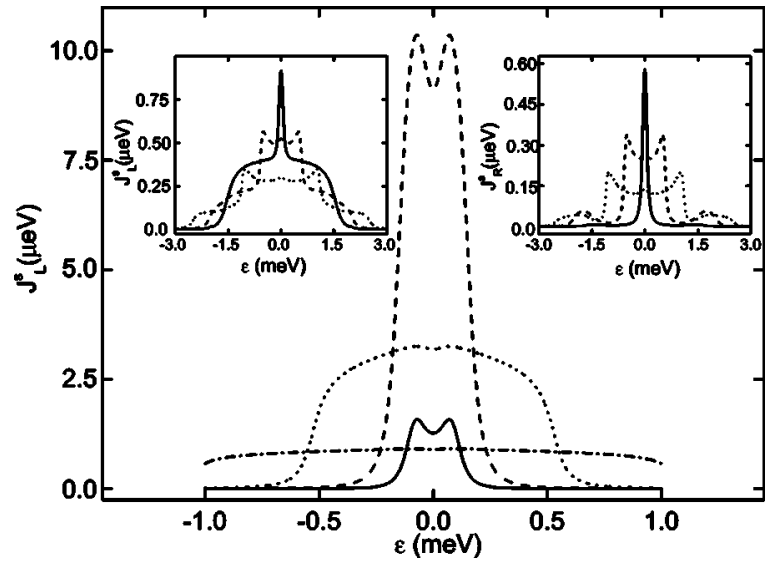

FIG. 6. $J_{L}^{S}$ vs $\epsilon$ at different $\omega: \omega=0.01 \mathrm{meV}$ (solid line), $\omega$ $=0.1 \mathrm{meV}$ (dashed line), $\omega=0.5 \mathrm{meV}$ (dotted line), and $\omega$ $=1.0 \mathrm{meV}$ (dash-dotted line). Other parameters are the same as Fig. 1. The right inset: $J_{R}^{S}$ vs $\epsilon$ at much larger frequencies: $\omega$ $=1.5 \mathrm{meV}$ (solid line), $2.0 \mathrm{meV}$ (dashed line), and $2.5 \mathrm{meV}$ (dotted line). The left inset: $J_{L}^{S}$ vs $\epsilon$ at different $\omega$. The symbols are the same as the right inset.

of them is dominated at low frequency. The other is present only for NS systems and is dominated at high frequency $\omega$ $>\Delta$. When the resonant level of the quantum dot is in line with the Fermi level of the lead, pure spin current is generated at finite frequencies with clear signature of resonance assisted pumping. The spin current may be detected in several ways: (1) When the spin current passes through a material with the spin-orbital coupling, there will be a charge imbalance which can be measured. ${ }^{36}$ (2) The electric field generated by the spin current ${ }^{37}$ can also be used to measure the spin current. (3) Recently, a method to measure the spin polarization of the current has been proposed and realized by Folk et al. ${ }^{38}$ using a gate-controlled bidirectional spin filter. With this technique, the measurement of spin current is experimentally feasible. In our theory, the rotating frequency of the field needs not to be large, and the device structure is quite typical; we believe the spin current should be experimentally measurable. In this paper, we did not consider the Coulomb interaction which may show additional interesting features. $^{39}$

\section{ACKNOWLEDGMENTS}

We gratefully acknowledge support by an RGC grant from the SAR Government of Hong Kong under Grant No. HKU 7032/03P, the CRCG grant from The University of Hong Kong, the grant from NSFC under Grant No. 10274052 (Y. D. W.), and the grant from NSFC under Grant No. 90303011 (B. G. W.). 
*Electronic address: jianwang@hkusub.hku.hk

${ }^{1}$ For recent reviews on spintronics, see, for example, S. A. Wolf, D. D. Awschalon, R. A. Buhrman, J. M. Daughton, S. von Molnar, M. L. Roukes, A. Y. Chtchelkanova, and D. M. Treger, Science 294, 1488 (2001); G. A. Prinz, ibid. 282, 1660 (1998).

${ }^{2}$ P. W. Brouwer, Phys. Rev. B 58, R10 135 (1998).

${ }^{3}$ M. Switkes, C. Marcus, K. Capman, and A. C. Gossard, Science 283, 905 (1999).

${ }^{4}$ F. Zhou, B. Spivak, and B. L. Altshuler, Phys. Rev. Lett. 82, 608 (1999).

${ }^{5}$ Y. D. Wei, J. Wang, and H. Guo, Phys. Rev. B 62, 9947 (2000).

${ }^{6}$ T. A. Shutenko, I. L. Aleiner, and B. L. Altshuler, Phys. Rev. B 61, 10366 (2000).

${ }^{7}$ Y. Levinson, O. Entin-Wohlman, and P. Wolfle, Physica A 302, 335 (2001)

${ }^{8}$ M. Moskalets and M. Buttiker, Phys. Rev. B 66, 035306 (2002); 68, 075303 (2003).

${ }^{9}$ M. Moskalets and M. Buttiker, Phys. Rev. B 66, 205320 (2002).

${ }^{10}$ O. Entin-Wohlman and A. Aharony, Phys. Rev. B 66, 035329 (2002).

${ }^{11}$ B. G. Wang, J. Wang, and H. Guo, Phys. Rev. B 65, 073306 (2002).

${ }^{12}$ J. L. Wu, B. G. Wang, and J. Wang, Phys. Rev. B 66, 205327 (2002).

${ }^{13}$ P. Sharma and C. Chamon, Phys. Rev. Lett. 87, 096401 (2001).

${ }^{14}$ E. R. Mucciolo, C. Chamon, and C. M. Marcus, Phys. Rev. Lett. 89, 146802 (2002).

${ }^{15}$ S. K. Watson, R. M. Potok, C. M. Marcus, and V. Umansky, Phys. Rev. Lett. 91, 258301 (2003).

${ }^{16}$ T. Aono, Phys. Rev. B 67, 155303 (2003).

${ }^{17}$ Y. D. Wei, L. H. Wan, B. G. Wang, and J. Wang, Phys. Rev. B 70, 045418 (2004).

${ }^{18}$ Z. Yao, H. W. C. Postma, L. Balents, and C. Dekker, Nature (London) 402, 273 (1999).

${ }^{19}$ K. Tsukagoshi, B. W. Alphenaar, and H. Ago, Nature (London) 401, 572 (1999).

${ }^{20}$ A. F. Morpurgo, J. Kong, C. M. Marcus, and H. Dai, Science 286, 263 (1999).

${ }^{21}$ D. H. Cobden, M. Bockrath, P. L. McEuen, A. G. Rinzler, and R.
E. Smalley, Phys. Rev. Lett. 81, 681 (1998).

${ }^{22}$ H. Mehrez, J. Taylor, H. Guo, J. Wang, and C. Roland, Phys. Rev. Lett. 84, 2682 (2000); H. Mehrez, H. Guo, J. Wang, and C. Roland, Phys. Rev. B 63, 245410 (2001).

${ }^{23}$ Y. D. Wei, J. Wang, H. Guo, and C. Roland, Phys. Rev. B 64, 115321 (2001).

${ }^{24}$ W. Zheng, J. L. Wu, B. G. Wang, J. Wang, Q. F. Sun, and H. Guo, Phys. Rev. B 68, 113306 (2003).

${ }^{25}$ A. Brataas, Y. Tserkovnyak, G. E. W. Bauer, and B. I. Halperin, Phys. Rev. B 66, 060404(R) (2002).

${ }^{26}$ B. G. Wang, J. Wang, and H. Guo, Phys. Rev. B 67, 092408 (2003)

${ }^{27}$ Q. F. Sun, H. Guo, and J. Wang, Phys. Rev. Lett. 90, 258301 (2003).

${ }^{28}$ W. Long, Q. F. Sun, H. Guo, and J. Wang, Appl. Phys. Lett. 83, 1397 (2003).

${ }^{29}$ R. Benjamin and C. Benjamin, Phys. Rev. B 69, 085318 (2004).

${ }^{30}$ When $\epsilon<0$, similar physics can be discussed using the picture of holes.

${ }^{31}$ It has been shown in Ref. 26 that for a two-dimensional quantum dot the orbital degrees of freedom does not contribute to the particle current in the presence of a rotating magnetic field.

${ }^{32}$ Y. Zhu, Q. F. Sun, and T. H. Lin, Phys. Rev. B 65, 024516 (2001).

${ }^{33}$ J. Wang, Y. D. Wei, B. G. Wang, and H. Guo, Appl. Phys. Lett. 79, 3977 (2001); J. Wang and B. G. Wang, Phys. Rev. B 65, 153311 (2002).

${ }^{34}$ Note that the total charge current is conserved so that the charge current of the right lead is equal to that of the left lead.

${ }^{35}$ Equation (48) can be shown mathematically from Eqs. (39)-(41) by changing $E$ to $-E$.

${ }^{36}$ J. E. Hirsch, Phys. Rev. Lett. 83, 1834 (1999).

${ }^{37}$ F. Meier and D. Loss, Phys. Rev. Lett. 90, 167204 (2003); Q. F. Sun, H. Guo, and J. Wang, Phys. Rev. B 69, 054409 (2004).

${ }^{38}$ J. A. Folk, R. M. Potok, C. M. Marcus, and V. Umansky, Science 299, 679 (2003)

${ }^{39}$ P. Zhang, Q. K. Xue, and X. C. Xie, Phys. Rev. Lett. 91, 196602 (2003). 